\title{
Spinal cord injury and autonomic dysreflexia: a case report
}

Apurva Bhatt, MSVI ${ }^{1}$, Brit Moore MD ${ }^{1}$, Talal Asif, MD ${ }^{1}$, Kristy E. Steigerwalt, PharmD, MLS ${ }^{1}$, Rebecca R. Pauly, MD ${ }^{1}$

\section{Author Affiliations:}

1. University of Missouri-Kansas City School of Medicine, Kansas City, Missouri

The authors have no financial disclosures to declare and no conflicts of interest to report.

\section{Corresponding Author:}

Rebecca R. Pauly, MD

University of Missouri-Kansas City School of Medicine

Kansas City, Missouri

Email:paulyr@umkc.edu 


\section{Abstract}

Autonomic dysreflexia (AD) is a life threatening condition affecting patients with spinal cord lesions T6 level and above. ${ }^{1}$ A 51-year-old male with history of paraplegia due to C6 spinal cord injury (30 years prior) presented with recurrent debilitating episodic diaphoresis, hypertension, low body temperature, and bradycardia. Previous hospitalizations presumed sepsis from UTI to be the etiology; however, on further evaluation his symptoms were consistent with undiagnosed $\mathrm{AD}$. This article describes a unique case presentation and reviews $\mathrm{AD}$ in depth, including the etiology, pathophysiology, and management.

\section{Keywords}

Autonomic dysreflexia, spinal cord injury, diaphoresis, hypertension, noxious stimuli

\section{Introduction}

Autonomic dysreflexia (AD) affects $48-90 \%$ of susceptible patients. ${ }^{2,3}$ Presentation of AD is consistent with sympathetic over-activation including hypertension, sweating, and headache, as well as paroxysmal bradycardia from intact parasympathetic input to the heart. AD is the result of noxious stimuli below the level of SCI inciting the sympathetic nervous system, which originates from T1-L2. With cervical and high thoracic SCI, the sympathetic spinal circuits are separated from normal supraspinal parasympathetic control, thereby causing unchecked sympathetic activity below the SCI. Parasympathetic activation of vagus nerve creates bradycardia in response to the hypertension caused by sympathetic activation of the splanchnic vasculature. 5 The most common noxious stimuli to provoke AD arise from bladder (over distension or infection) or bowel (fecal impaction), but can also occur with abdominal infections (appendicitis, cholecystitis, and pancreatitis), decubitus ulcers, and fractures. ${ }^{4}$ Complications of $\mathrm{AD}$ arise from acute uncontrolled hypertension with resultant end organ damage such as stroke or myocardial infarction. ${ }^{6}$

\section{Patient Case}

A 51-year-old male with history of C6 SCI with subsequent paraplegia, neurogenic bowel/bladder, frequent urinary tract infections, osteoporosis, and myalgia presented to his primary care physician with a two-day history of severe sweating and increasing lower extremity spasticity. Medical records revealed numerous clinic visits, emergency department visits, and 
hospitalizations for similar symptoms. He was treated for urinary infections without the etiology of extreme diaphoresis or hypertension being determined. He refused self-catheterization home bladder management due to fear of urethral trauma. He employed Crede's maneuver of applying suprapubic pressure to aid in urination when he 'felt the urge to urinate'. ${ }^{6}$

The patient complained of chills, severe sweats, anxiety, and difficulty urinating. He denied change in urine color or malodor. On presentation, he was tremulous and diaphoretic above the nipple line, requiring use of a towel to wipe excessive perspiration from his face and neck. Vital signs showed hypertension of $170 / 100$, low oral temperature of $95.4^{\circ} \mathrm{F}$ and bradycardic heart rate of 49 beats/minute. Neurological exam revealed known sensorimotor deficits (insensate below T4 dermatome, 0-1/5 strength throughout lower extremities) with increased spasticity in lower extremities from baseline. Skin exam revealed no decubiti. Urinalysis showed large leukocyte esterase, white blood cells, negative nitrite, $3+$ bacteria, and 0-2 squamous epithelial cells. Postvoid residual bladder scan volume was elevated at $249 \mathrm{ml}$.

Tight clothing was removed and a loose fitting hospital gown placed. Intravenous normal saline was started with empiric antibiotics (piperacillin-tazobactam) while cultures were pending. He strongly expressed the desire not to have bladder catheterizations (which we honored) due to previous complications. Within five hours of admission, blood pressure improved to 125/80 and temperature normalized to $97.6^{\circ} \mathrm{F}$. On day two, patient's presenting complaints of elevated blood pressure and diaphoresis had resolved. Blood cultures remained with no growth. Urine culture grew E. Coli sensitive to trimethoprim-sulfamethoxazole to which he was converted. Prior to discharge, patient was educated about $\mathrm{AD}$ as the cause of his episodic excessive sweating, the potentially life threatening associated hypertension, and counseled on techniques to avoid bladder over distension. Follow up with urology clinic for urodynamic studies and with primary care physician for continued care were arranged.

\section{Discussion}

This case report demonstrates the importance of recognition and immediate treatment for patients with SCI and AD. This patient had unrecognized clinical signs and symptoms consistent with $\mathrm{AD}$ on prior medical encounters. This admission provides three possible inciting causes for AD: noxious stimuli from cystitis, chronic bladder over distension, and irritation of tight clothing. His

hypertension, bradycardia, low body temperature, and diaphoresis are features we will discuss in more detail.

Understanding the pathophysiology of AD is crucial to management. Noxious stimuli below level of SCI increase sympathetic outflow from the spinal cord. Regional vasoconstriction leads to a marked rise in arterial blood pressure. There is loss of descending modulation of sympathetic activation from the parasympathetic nervous system due to signal interruption at the level of the 
SCI. Normally the parasympathetic nervous system stimulates splanchnic bed vasodilation to reduce systemic blood pressure. The only parasympathetic modulation present with $\mathrm{AD}$ is baroreceptor mediated activation of vagus nerve in response to hypertension, with resultant bradycardia and vasodilation above the SCI. This response alone, however, is typically not enough to counteract increased blood pressure from unchecked sympathetic activity. This patient's heart rate of 49 is consistent with this mechanism.

This patient presented with low body temperature, which is thought to be secondary to thermoregulatory dysfunction. ${ }^{7,8}$ The literature describes a patient presenting with hypothermia associated with $\mathrm{AD}$ which is in contrast to prior reports of temperature elevations. ${ }^{7}$ The case hypothesizes that excessive sweating in association with thermoregulatory dysfunction contributes to the significant hypothermia experienced by patients with AD. ${ }^{7}$ Abnormal thermoregulatory control is due to reduced sensory input to thermoregulatory centers and loss of sympathetic control of temperature and sweat regulation below the level of injury. ${ }^{8}$ Low body temperature as exhibited by our patient is an important feature of AD.

Management of $\mathrm{AD}$ includes 1) sitting upright and loosening all tight fitting clothing and devices (elastic band from urine leg bag, TED hose, abdominal binder); 2) identifying and removing noxious stimuli (evaluating for bladder infection, bladder distension, fecal impaction, etc.); 3) monitoring vital signs every 2-5 minutes during the episode; and 4) observing for recurrent symptoms for at least 2 hours after the time of improvement. Rapidly acting medications such as IV labetalol that prompt reduction of blood pressure may be needed depending on severity. ${ }^{1}$

Normal bladder storage is predominantly a sympathetic response. Internal sphincter contraction and bladder wall relaxation promote urine storage. Normal bladder emptying is predominantly a parasympathetic response. Normal bladder capacity is $300-600 \mathrm{~mL}$. Patients with SCI can have aberrant sympathetic stimulation of their bladder secondary to noxious stimuli. This increased sympathetic stimulation promotes bladder storage, which was observed in our patient with a post void residual of $249 \mathrm{ml}$, despite his use of the Crede's maneuver.

Close monitoring of bladder health is crucial for patients with SCI and urodynamic studies should be performed to assess function of the bladder neck, external sphincter, and detrusor muscle. It is also important for patients to maintain bladder volumes below $500 \mathrm{ml}$ to avoid vesicoureteral reflux, overflow incontinence, or hydro-ureter. Regularly scheduled timed voids decrease the risk of bladder distension and bacterial overgrowth. Techniques such as the Crede's maneuver can improve urinary voiding in certain cases. ${ }^{6}$ Medications such as alpha-adrenergic receptor blockers like tamsulosin or cholinergic stimulators such as bethanechol may be useful. In patients unable to void spontaneously, the use of regularly timed intermittent catheterization (IC) throughout the day is advised. IC is preferred to indwelling catheters due to fewer complications (epididymitis, urethritis, strictures and recurrent urinary tract infections). This 
patient did not seek regular care with urology and did not complete scheduled urodynamic studies. Urodynamic studies in this patient could have identified preventable causes of his AD.

Methods to reduce the risks and symptoms of AD were discussed in detail with our patient including use of regularly scheduled voids, techniques to assist in more complete bladder emptying, medications to assist in more complete bladder emptying, and use of IC when necessary to decrease risk of bladder over distension. As AD can be life-threatening, early recognition of symptoms, signs, and provoking noxious stimuli as well as prompt treatment as outlined in this case report are critical in preventing morbidity and mortality of this vulnerable patient population. 


\section{References}

1. Bycroft J, Shergill IS, Chung EAL, Arya N, Shah PJ. Autonomic dysreflexia: a medical emergency. Postgrad Med Journal. 2005;81(954):232-5.

2. Kirshblum S, Gonzalez, P, Nieves, J, Cuccurullo S, Luciano. Spinal cord injuries. In: Cuccurullo, S, editor. Physical medicine and rehabilitation board review. 2nd ed. New York NY: Demos Medical Publishing; 2010. pp. 562-73.

3. Phillips AA, Krassioukov, AV. Contemporary cardiovascular concerns after spinal cord injury: mechanisms, maladaptations and management. J Neurotrauma. 2015;32:1927-42.

4. Shergill IS, Arya M, Hamid R, Khastgir J, Patel HR, Shah PJ. The importance of autonomic dysreflexia to the urologist. BJU Int. 2004;93:923-6.

5. Karlsson AK. Autonomic dysreflexia. Spinal Cord. 1999;37:383-91.

6. Cruz ES, Stolzenberg D, Moon D, Maitin IB, Cruz E. Medical emergencies in rehabilitation medicine. In: Maitin IB Cruz E editors. Current Diagnosis \& Treatment: Physical Medicine \& Rehabilitation. New York NY: McGraw-Hill; 2015. pp. 96,587.

7. Colachis III, Sam C. Hypothermia associated with autonomic dysreflexia after traumatic spinal cord injury. American Journal of Physical Medicine \& Rehabilitation. 2002;81(3):232-5.

8. Hagen, EM. Acute complications of spinal cord injuries. World Journal of Orthopedics. 2015;6(1):17-23. 